\title{
Gestión de dinámicas de grupos en la enseñanza de ELE a adultos en contextos de instrucción formal: una pieza más del complejo fenómeno del aprendizaje
}

\author{
María del Carmen Méndez Santos \\ Universidad de Alicante \\ macms@ua.es \\ @mariaparrula
}

\begin{abstract}
Resumen: En este artículo se realiza una revisión teórica sobre la importancia de las dinámicas de grupo como un elemento más del sistema dinámico complejo que es el proceso de enseñanza-aprendizaje de una lengua extranjera. En primer lugar, se traza la trayectoria histórica teórica y se perfila también la relación de las dinámicas de grupo con otros aspectos como la motivación, la exposición al input o la mejora de aspectos afectivos. Posteriormente, se describe qué es un grupo y cómo se caracteriza para, por último, ofrecer reflexiones didácticas, constatar tendencias y proponer ejemplos de actividades de clase.
\end{abstract}

Palabras clave: español como lengua extranjera, instrucción formal, grupo, aprendizaje. 


\title{
Management of group dynamics in teaching adults in formal instruction contexts: one piece more of the complex learning phenomenon
}

\begin{abstract}
In this paper, a theoretical review is carried out on the importance of group dynamics as another element of the complex dynamic system that is the teaching-learning process of a foreign language. First, a theoretical historical trajectory is drawn up and about the relationship of group dynamics with other aspects such as motivation, exposure to input or improvement of affective aspects. Subsequently, the term group is described and its characteristics in order to, finally, offer didactic reflections, substantiate trends and propose examples of class activities.
\end{abstract}

Key words $¥$ Spanish as a foreign language, formal instruction, group, learning.

Recibido el 16/05/2018

Aceptado el 24/09/2018 


\section{Introducción}

La enseñanza formal de lenguas extranjeras a adultos puede producirse en tantos contextos como podamos imaginar: centros educativos públicos o privados con aulas dotadas de la última tecnología y grupos reducidos o centros cuyos recursos disponibles son escasos y la ratio de estudiantes, mayor. No obstante, en todas esas situaciones de aprendizaje podemos identificar un denominador común: el factor humano. Visualizamos al profesorado y al grupo de alumnos. Entre esas personas se establecen unos vínculos que constituyen un nódulo más de un sistema complejo de factores que componen la experiencia de enseñanza-aprendizaje de una lengua extranjera (LE, en adelante). En otras palabras, la dinámica del grupo afecta, se adapta y trabaja junto con todos los otros factores que dan lugar al fenómeno de enseñanza-aprendizaje, pero no es el único ni el más determinante, sino que todos trabajan en una sinergia.

Para evitar el reduccionismo que en la tradición investigadora implicaba el analizar un solo elemento como el único factor condicionante de todo lo demás y en torno al cual pivotaba el resto del sistema, las últimas propuestas teóricas ofrecen un nuevo marco que entiende el fenómeno de enseñanza-aprendizaje desde una perspectiva holística donde todos los factores implicados (profesorado, alumnado, método, materiales, horario, motivaciones, ...) interactúan, se adaptan y cambian a lo largo del tiempo. Por visualizarlo, una metáfora que podría describir el proceso de interdependencia de esos elementos implicados sería un juguete móvil de madera en el que cuando se toca, siquiera se roza, una pieza, el sistema entero entra en movimiento. Las consecuencias de ese cambio no son predecibles y estas dependen de la intensidad, del tiempo y del tipo de perturbación (Hiver, 2015: 23) realizada. El tener en consideración esas múltiples relaciones interdependientes de los factores implicados, nódulos si se sigue el marco conceptual de redes (Mercer, 2015: 75), parece ser capaz de dar una mejor explicación a algunos temas de debate sobre la enseñanza de una LE, ya que el adjudicar todo el éxito o todo el fracaso del aprendizaje a solo un factor, como decíamos, no parecía ajustarse a la realidad del aula.

Además de la interdependencia de esos elementos y la no linealidad, esta concepción teórica, como describe Larsen-Freeman (2015: 11 y ss.), toma como central el concepto del cambio y de emergente. En consecuencia, los elementos implicados en ese fenómeno (alumnado, profesorado, método, motivaciones, actividades...) se afectan entre sí de modo que emerge un sistema nuevo en el que todos coaparecen, en este caso, el proceso de enseñanza-aprendizaje. Asimismo, este sistema se caracteriza por ser cambiante a lo largo del tiempo, en función 
de los inputs a los que se enfrenta y cómo estos modifican a todos los elementos presentes.

Esta teoría general, adaptativa, interdependiente, cambiante y no linear circunscribe el fenómeno de la enseñanza-aprendizaje como un sistema dinámico complejo. Así se nos ofrece una panorámica más comprensiva de las fluctuaciones que suceden entre y dentro de los elementos que componen el aprendizaje. De este modo, cabe la posibilidad de explicar por qué una perturbación del sistema puede tener una fuerte consecuencia o no en función del tiempo de exposición, por ejemplo, como podría ser el caso de los efectos que tendría una actividad que no funciona o un momento de incomprensión o frustración del alumnado. El impacto de esa perturbación se ve absorbido por los distintos elementos del sistema y en función de su fuerza y tiempo de permanencia el sistema se reorganiza (con éxito o no).

Por todo ello, no se debe entender el análisis de los procesos de enseñanzaaprendizaje de una LE como algo reducible a una serie de hechos aislados que funcionan como una receta mágica en cualesquieras circunstancias. Esa perspectiva de trabajo ha hecho naufragar durante años enfoques, métodos y fórmulas que "funcionaban", pero que a la hora de replicarse no tenían éxito debido a la ingente cantidad de circunstancias que afectan a cada sistema y que eran obviadas para generalizar y soslayar cada caso concreto de enseñanza-aprendizaje.

En particular esta nueva forma de abordar la realidad se ha aplicado a la investigación sobre motivación en el campo de la enseñanza de LE dando paso así a un nuevo ciclo teórico conocido como el período sociodinámico (Kikuchi, 2015: 21) donde la dinámica de grupos también es considerada como una parte del sistema. Esta nueva etapa viene a superar las limitaciones de las tres anteriores conocidas como social-psychological period, cognitive-situated period y process-oriented period. En la primera de ellas, se encuentran las teorías que triunfaron entre los años 60 y 90 de las que Gardner y Lambert (1972) fueron los máximos exponentes. Estos autores se centraron en las actitudes que tenían los aprendices hacia la comunidad de la lengua meta, ya que su contexto de trabajo era Canadá. Ellos definieron dos conceptos que han dirigido la concepción de la motivación durante décadas: orientación integradora y orientación instrumental. No obstante, como señala Dörnyei (2008:38, 2009), en los años 90 los investigadores quisieron salvar la distancia real que había entre esos estudios generales de la psicología y el aula de enseñanza de una L2, ya que se habían minimizado la importancia de otros aspectos que concurren en el aula, entre ellos la dinámica del grupo. Esta transformación se conoce como el período cognitive-situated y abarca la década de los 90. Así surgieron teorías como las de la eficacia personal (Bandura, 1997) que 
se centra en la "valoración" que hacen las personas sobre sus capacidades para aprender; la de la atribución de Weiner (1992), que giraba en torno a la idea de sobre quién recae la responsabilidad de los éxitos o los fracasos del aprendizaje; la de Deci y Ryan (1985) sobre la "autodeterminación" o la motivación intrínseca y extrínseca, y otras tantas. No obstante, como señala Dörnyei (2008: 33):

Las teorías puras de la motivación, es decir, los modelos que representan una única perspectiva teórica, y que por tanto están anclados en unos cuantos factores de motivación pero que prácticamente ignoran los estudios que sigan otras líneas de pensamiento, no son aplicables en el aula de manera eficiente.

Esta ineficacia se debía a que se ignoraban el resto de los factores sobre los que no se ponía "la lupa" y se desatendía, además, los cambios que se producían a lo largo del tiempo en la vida real. Así, en el período de cambio de siglo la perspectiva general de trabajo dejó de ser "unifactorial" y "estática" para centrarse en una perspectiva más global y dinámica como la que dibujaron Williams y Burden (2008:146) y Dörnyei y Otto (1998:48). Era global porque en sus análisis tenían en cuenta aspectos internos como el interés intrínseco por la actividad, el valor percibido de la actividad, el sentimiento de agencia que se tiene, el grado de dominio, el autoconcepto, las actitudes que se tienen, otros estados afectivos que se experimentan, la edad y el sexo. Entre los factores externos destacaban las personas significativas para el aprendizaje, el carácter de la interacción con otras personas significativas, el entorno y el contexto más amplio en el que se da la enseñanzaaprendizaje. La conjunción de todos estos elementos, internos y externos, y la dependencia que entre ellos se da dibujaba un panorama del grado de motivación mucho más rico que el hecho de tener en cuenta solamente uno de ellos. Además, entendían que la motivación era algo dinámico, procesual, cambiante. Es en estos años cuando los estudios de psicología se han reorientado hacia constructos más flexibles y adaptativos que desembocan en la actual concepción conocida como "período socio-dinámico" que consiste, como hemos explicado, en un enfoque más holístico que tiene en consideración la interrelación de diferentes elementos o condiciones relevantes para la situación de aprendizaje a través del tiempo y teniendo en cuenta el ecosistema en que se producen, más que analizar las relaciones de los factores de forma aislada y única (Dörnyei, MacIntyre y Henry, 2015: 1).

Será en este marco en el que nos situamos y, por ello, todo lo que aseveremos sobre la importancia de la dinámica de grupo no debe ser descontextualizado del resto de los factores con los que interactúa y con los que conjuntamente puede repercutir para alcanzar unas mejores condiciones de aprendizaje. Entenderemos pues la dinámica de grupo como un estado atractor (Hiver, 2015: 23) más de este 
sistema dinámico complejo y que ejerce por ello una influencia sobre los demás. En el caso de interactuar armónicamente esta puede ayudar a sobrellevar el impacto de posibles perturbaciones negativas. Es más, ante un estado neutro inicial consideramos que una buena dinámica de grupo puede ejercer como un fuerte atractor para ser capaz de mantener en un estado positivo otros de los factores implicados y ayudar a sobrellevar atractores negativos débiles. En otras palabras, una buena dinámica de grupos puede crear una red de soporte de emergencia ante casos de desmotivación puntual, por ejemplo, ante una actividad que no funciona o no gusta a todo el mundo. Ese fuerte atractor puede ayudar a sobrellevar la presencia de las discordancias menores que se dan día a día en el aula.

\section{El papel de la dinámica de grupos en el proceso de enseñanza-aprendizaje de ELE}

Partiendo de la base de que el análisis de los factores implicados en el proceso de enseñanza-aprendizaje debe ser visto de un modo global e interdependiente que fluctúa a lo largo del tiempo, vamos a analizar cómo la dinámica de grupos se conecta con otros aspectos. Para ello describiremos primero cómo se ensambla dentro de un marco educativo teórico más general y de la investigación en lingüística aplicada en particular. Asimismo, buscamos delinear tendencias extraídas de experiencias exitosas llevadas al aula para poder ofrecer consejos prácticos para el profesorado de español como lengua extranjera (en adelante, ELE).

\subsection{Marco teórico sobre la importancia de los grupos}

Es en un marco general en el que entendemos que la dimensión psicológica del ser humano tiene un aspecto social y que "el hecho de tener amistades y disfrutar de la compañía es una importante fuente de bienestar y salud" (Oblitas, Martínez Sánchez, Palmero; 2008:349). Esto redunda en una implicación pedagógica clara que recogía, por ejemplo, Vigotsky y es que el aprendizaje "estimula y activa una variedad de procesos mentales que afloran en el marco de la interacción con otras personas, interacción que ocurre en diversos contextos y es siempre mediada por el lenguaje" (Carrera y Mazzarella, 2001: 43). Esta perspectiva ve recogido su espíritu en el conocido como "interaccionismo social" cuya aplicación a la enseñanza de LE se materializa en que un estudiante aprende cuando tiene la ocasión de usar la lengua a través de "interacciones significativas y, por tanto, participa en la construcción de su propio conocimiento y comprensión de la lengua” (Martín Perís, 2008). Emerge en este punto un concepto fundamental para la enseñanza de una LE que es el de "interacción". 
Si tenemos en cuenta las teorías del procesamiento de la información, entendemos que la interacción oral interpares puede proporcionar contextos significativos que favorecen el aprendizaje, pero sobre todo concebimos que esa interacción conllevara gran cantidad de output producido.

. Este aspecto, según la hipótesis del output de Swain (1993), es determinante en el proceso de aprendizaje porque lo que falta para conseguir el éxito no es tanto tener mucha exposición al input, como lograr una mayor producción: "What was lacking was sufficient opportunities for language use" (Gass y Mackey, 2015: 183). Así, el promover la interacción interpares con frecuencia puede llegar a paliar la falta de exposición en contextos de no inmersión, ya que el tiempo es limitado y con frecuencia se reduce a unas horas de clase a la semana (De Santiago y Fernández, 2017:792). Por otro lado, esta intensa actividad de producción oral que se puede promover en clase puede influir también en el nivel de automatización de la lengua, es decir, en la rutinización del uso del lenguaje a través de muchos intentos (Gass and Mackey, 2015: 185).

Por todo lo expuesto, entendemos que la dinámica de grupos, como un atractor más del sistema dinámico complejo, repercute en aspectos cognitivos como la recepción de input y la producción de output por el papel activo que el alumno debe tener (Stevick, 1980:80), así como que redunda en mayores niveles de motivación ante actividades que fomenten la seguridad y la cohesión grupal, así como la autoestima de los aprendientes, entre otras consecuencias de la interdependencia de factores que podrían citarse. En otras palabras, la dinámica de grupos afecta a todos los nódulos de la red tanto a aspectos cognitivos, como metodológicos, afectivos y socioculturales.

\subsection{Caracterización de los grupos}

Podemos definir grupo como "a collection of interacting people with some degree of reciprocal influence over one another" (Wlodowski, 1985: 197). Cada grupo es único y genera sus relaciones, reglas, rutinas y límites. La dinámica que entre las personas se establece será el "resultado del contacto entre los estilos interactivos del profesor y de los alumnos, las experiencias que surgen sobre la marcha en el proceso y los agrupamientos que se hayan constituido para facilitar la enseñanza y el aprendizaje" (Richards y Lockhart, 2008: 142). Así pues, la primera consecuencia didáctica que debemos tener clara es que es necesario reflexionar sobre lo que ocurre en el aula, en otras palabras, entender cómo funciona el grupo para potenciar o mejorar sus relaciones en la medida de lo posible. Esta capacidad se encuentra registrada como una de las competencias clave que el profesorado de ELE debe desarrollar (Instituto Cervantes, 2012), ya que se requiere que el 
docente "fomente un espacio seguro en el que todos participen y trabajen en colaboración" y debe "dinamizar el grupo seleccionando el tipo de agrupamiento más adecuado para cada actividad, asignando roles a los alumnos cuando convenga, $(\ldots)$, etc.

Un grupo eficaz y donde el aprendizaje "fluye" puede surgir de una forma espontánea o puede ser el resultado de una planificación proactiva y pormenorizada. Entendemos por flujo ese "estado en el cual las personas se hallan tan involucradas en la actividad que nada más parece importarles; la experiencia por sí misma es tan placentera que las personas la realizarán incluso aunque tenga un gran coste por el puro motivo de hacerla" (Csíkszentmihályi, 2014: 16). Para alcanzar ese estado de aprendizaje prodigioso la relación entre las personas de la clase no puede ser tensa y por ello debe lograrse una "armonía interpersonal" (Goleman, 2014: 13) que garantice que los integrantes coordinan sus esfuerzos para ser más productivos. Lo que se conoce como convertir el capital bumano en capital social (Mercer, 2015: 76). Esa dinámica armónica debe conseguirse a través un trabajo de largo recorrido desde la primera clase hasta la última, teniendo en cuenta qué fluctuaciones suelen darse en el tiempo, y debe comenzar con una estrategia clara para establecer la filiación al grupo, desarrollar actitudes positivas, enriqueciendo las actividades haciéndolas significativas y fomentando la sensación de competencia de los aprendices. Dada esta importancia parece poco práctico dejar al azar que la dinámica aparezca espontáneamente y por ello defendemos la necesidad de reflexionar sobre técnicas que pueden resultar útiles en combinación con otras estrategias.

La cohesión grupal, en otras palabras, el sentimiento de filiación respecto a un grupo, conviene cultivarla desde el primer día. El objetivo es contribuir a que el ambiente de clase haga sentir tanto al alumnado como al profesorado que son respetados y que están conectados entre sí (Ginsberg y Wlodkowski, 2000: 6). Promover este sentimiento hará que los miembros perciban que es mejor permanecer en el grupo que dejarlo (Wlodkowski, 1985: 198), ya que si se logra alcanzar esa cohesión grupal, la experiencia de aprendizaje se vive con una actitud positiva (Clement, Dornyei y Noels, 1994: 442).

¿Qué implicaciones conlleva esa actitud positiva? En primer lugar, un menor grado de ansiedad ${ }^{1}$, ya que los estudiantes con ansiedad no se comunican tanto como los alumnos relajados (MacIntyre, 2017: 17) y si un aprendiz no se siente parte del grupo deja de hacer esfuerzos por comunicarse. Esto, como ya hemos

1. Conviene recordar que, según King y Smith (2017: 104), cierto grado de ansiedad social es natural, ya que aparece en tareas de actuación social, como hacer presentaciones, por ejemplo. No obstante, la circunstancia preocupante es cuando ese grado de ansiedad inhibe el aprendizaje porque provoca que los estudiantes no sean capaces de hacer la actividad. Ese es el momento en que se requiere una intervención del profesorado. 
explicado, repercute negativamente en otro nódulo del sistema como es la producción de output que es crucial (Swain, 1993) para avanzar en la interlengua. En segundo lugar, se crea un ambiente emocional de valoración, respeto y cooperación sin miedo a ser juzgado, por lo que la posibilidad de cometer errores y el miedo a perder prestigio se reducen y esto promueve la seguridad en sí mismos de los estudiantes y aumenta su autoestima (Clement, Dörnyei y Noels, 1994: 442). Además, como indican Hadfield (1992: 10) y Dörnyei (2001: 40), los grupos cohesionados incrementan su productividad y, por lo tanto, mejoran sus resultados de aprendizaje.

Además de la cohesión y de promover ese sentimiento positivo, es necesario enriquecer las actividades haciéndolas realmente significativas y consiguiendo que los estudiantes sean conscientes de su valor y competencia. Apoyar el sentimiento de optimismo y esperanza sobre el aprendizaje hace que, como recoge Goleman (2014: 239) se incremente el sentimiento de "autoeficiencia". Este estado de alto grado de percepción de la competencia tiene consecuencias positivas en la autoestima y en el grado de valía personal percibida (Covington, 1998: 15).

Si se opta por una actitud proactiva, conviene tener claro que todo esto no se logra en un día ni es fruto de una sola estrategia que incide en un solo factor. Desde el comienzo del curso es necesario implementar técnicas que fomenten esa filiación, cohesión, autoeficacia, alta autoestima, etc. Esta actitud y compromiso deben mantenerse a lo largo de las clases y terminar con una fase de reafirmación y autoevaluación positiva (Dörnyei, 2008: 43).

Por otra parte, y como ya hemos indicado, la idea de proporcionar actividades "significativas" es fundamental. Esto puede conllevar un cambio de algunas estrategias didácticas, ya que se debe otorgar al alumnado "una función activa, puesto que ha de construir sus conocimientos, reajustándolos a su sistema cognitivo. El profesor tiene la función de proporcionar información nueva comprensible y significativa, compatible con los conocimientos que el aprendiente ya posee" (Martín Peris, 2008). Por todo lo explicado, no es posible entender la dinámica de grupos como algo que solo se trabaja en una actividad o sesión, sino que forma parte de una sinergia dada entre un grupo abierto de componentes del sistema. Es una actitud global que debe abarcar diversas estrategias didácticas que se compatibilicen con el desarrollo de la dimensión cognitiva, social, cultural y lingüística. 


\section{Implicaciones didácticas de la gestión de la dinámica de grupos}

Como se explicaba anteriormente, este trabajo es una reflexión teórica que intenta extraer tendencias de experiencias de aula exitosas con el fin de proporcionar ciertas ideas que puedan ser aplicadas y adaptadas a otros contextos de aprendizaje. Nos situamos en un nivel temporal sobre las diferentes etapas fundamentales en el desarrollo de los grupos: inicio, desarrollo y disolución. No vamos a ahondar en esta ocasión en el nivel del día a día por cuestiones de limitaciones de espacio.

Los grupos de estudiantes con los que se ha trabajado de esta manera tienen en común que son alumnado universitario de centros públicos, es decir, adultos que cursan estudios de grado que incluyen en su currículum el español. En el caso de los aprendices que formaban parte de las clases en la Universidade de Vigo se trataba de estudiantes de intercambio, mayoritariamente europeos que tomaban los cursos de lengua de manera opcional, por lo que los grupos eran multilingües, de diferentes estudios y nacionalidades. En el caso de los discentes japoneses ellos tenían las clases como parte de su carrera, ya que cursaban estudios de lenguas extranjeras y el español era su lengua principal. Los grupos rondaban la cifra de los veinte estudiantes y el horario de las clases variaba, pero cierto es que no eran en ningún caso los más deseables: primera hora de la mañana en Japón y justo después de la comida en el caso de las clases desarrolladas en España. La mayoría de los aprendices en ambos casos eran mujeres, aunque el porcentaje de hombres rondaba el 20\%. En el caso de Japón se reflexiona sobre prácticas y estrategias llevadas a cabo en tres grupos de nivel inicial y en el caso de España, de varios grupos de A1 y A2. Esta descripción sucinta del perfil y del contexto no pretende ser exhaustiva porque no se trata de estudios de caso, sino, como se explicaba, de una abstracción de técnicas, estrategias y sesiones que han sido repetidas en diferentes contextos de aula a lo largo de varios años y que se han mostrado eficaces para mejorar la dinámica del grupo y el aprendizaje en general.

\subsection{Los primeros días}

Durante las primeras sesiones el profesorado debe ser un modelo de actitud positiva transmitiendo claridad sobre las normas, el modo de trabajo que se va a usar y los objetivos que se buscan, ya que "casi todo lo que hace un profesor en el aula influye en la motivación de los alumnos" (Dörnyei, 2008: 56).

Es conveniente que la primera impresión haga sentir al alumnado seguro, exitoso, interesado y que lo aprendido tenga relevancia personal (Gingsberg y 
Wlodkowski, 2000ः 81) ¿ ¿Cómo es posible operativizar estos objetivos en el aula en las sesiones iniciales? En primer lugar, para alcanzar una zona de seguridad emocional (Dörnyei, 2008: 70) donde se sientan tranquilos conviene minimizar, sino eliminar, la exposición pública negativa, como, por ejemplo, la que se hace cuando se corrigen los errores individualmente y personalizándolos, dado que esto puede provocar desmotivación, ansiedad, afectar a su autoestima, perjudicar su imagen pública, etc. (Oxford, 2000: 84). Por otra parte, ¿cómo es posible lograr que algo sea relevante para todos? Parece que la única respuesta viable es la posibilidad de elegir, ya que lo que puede resultar motivante para unos aprendices puede ser desmotivante para otros porque los individuos varían en sus percepciones (Kikuchi, 2015: 22). El poder elegir, por ejemplo, el tema de trabajo ofrece una flexibilidad y un alto grado de relevancia personal. Además, en esas primeras jornadas, en la mayoría de las ocasiones, la gente no se conoce y puede sentir suspicacia, inseguridad, incertidumbre, etc. Las personas evitan quedar en evidencia y analizan el desarrollo y los roles que otros estudiantes van tomando (Dörnyei y Malderez, 2000: 177). Por ello, en este tiempo deben establecerse las normas del grupo, clarificar los objetivos y crear las relaciones entre los miembros, ya que estas pueden mejorar la calidad del esfuerzo del alumnado, la moral y los logros académicos (Ehrman y Dörnyei, 1998). Las normas pueden ser las culturales, las institucionales o las particulares del aula. En lo que atañe al aspecto institucional y académico es necesario explicitar y dejar claras las cuestiones administrativas o funcionales respecto al curso (matrículas, períodos de examen, ...) y mejor por escrito en un lugar al que puedan acudir en todo momento. En lo que respecta a las normas culturales es importante tener en cuenta que el alumnado puede no conocerlas o no ser conscientes de esa dimensión cultural que tiene comunicarse con otros compañeros del aula, en el caso de ser aprendices provenientes de diferentes orígenes. Aunque pudiese parecer obvio, "a pesar del crecimiento del turismo y comercio internacionales", los estudiantes pueden "dar muy poco por sentado" (Byram y Fleming, 2001: 18). Así pues, una propuesta didáctica en los primeros días para evitar malentendidos que podrían crear un ambiente hostil podría centrarse en las normas culturales de diferentes países respecto a las clases.

Por ejemplo, para esos primeros días se podría llevar a cabo el siguiente modelo de sesión (o parte de una sesión, siempre en función de las circunstancias y adaptándolo al sistema de aprendizaje concreto al que uno se enfrenta), desde el nivel A2 (o sesión de repaso de pasados de B1), que combina aspectos léxicos, funcionales, culturales y gramaticales de una forma integrada. Se podría comenzar la sesión con una serie de fotos de escuelas del mundo y estudiantes diseminadas por las mesas o paredes (o proyectadas en una pantalla). Esto tendrá mejores 
resultados en grupos de múltiples nacionalidades y regiones. El alumnado deberá moverse e intentar identificar el país al que pertenecen y poner etiquetas a los objetos que reconocen. Esta tarea se desarrolla en unos 5 o 10 minutos. Con esta dinámica conseguimos activar el vocabulario sobre aspectos académicos que suele aparecer en las primeras unidades de los libros de A2, hacerlo más visual y comunicativo, ya que cuando se encuentren ante las fotos los aprendices tenderán a hablar, comentar, hacerse preguntas, formular hipótesis, cooperar para nombrar los objetos, etc. Una vez que hayamos controlado que todo el mundo ha participado, les pediremos que se agrupen en torno a aquellas fotos que les hayan llamado más la atención. Verbalizamos esta instrucción en plural para que, si necesitamos recolocar a alguien para compensar los grupos, esto sea "percibido" como algo aceptable y que no se haga a regañadientes. Normalmente siempre hay alguien más "dispuesto" en caso de necesidad a reagruparse para que sean números equilibrados. Pondremos tantas fotos como grupos queremos que se formen, calculando que el tamaño medio debería rondar las 4 o 5 personas, ya que esto garantiza mayores oportunidades de que todos los estudiantes puedan hablar. Wlodkowski (1985: 206) sitúa la cantidad adecuada en un rango de unas 2 a 6 personas y Richards y Lockhart (1998: 43) entre 2 y 4. Lo que queda claro, en todo caso, es que los grupos deben ser reducidos para evitar la ansiedad que provoca "romper" a hablar en un gran grupo (Horwitz, 2017: 43) ${ }^{2}$.

Después, para cada equipo habrá una ficha en la que deben escribir sus hipótesis sobre la duración del año académico, duración de las clases, qué días de la semana hay clases, normas de clase, si les mandan deberes para casa, si se estudia español u otros idiomas en las escuelas... Deberán discutir y cooperar para contestarlas. Si en un grupo no hay interacción porque no conocen ninguna de las respuestas se les puede invitar a buscar en internet más información. Procuraremos elegir algunas imágenes que les resulten familiares y otras más "exóticas". Después de aproximadamente 15 minutos y no más de 20, o cuando hayan acabado si es antes, les pediremos una puesta en común de los datos obtenidos y los apuntaremos en la pizarra. Esto nos llevará unos 15 o 20 minutos. Repasaremos contenidos léxicos en función el nivel del curso. Prestaremos atención especial a las preguntas relacionadas con la presencia del español en la educación y a las normas de clase. Entonces les preguntaremos qué se tolera y qué no según cada país (contenido gramatical: se puede/no se puede, está permitido/está prohibido). Volverán a participar todos en sus grupos contando las normas de sus diversos países y recordando sus experiencias anteriores estudiando y en particular idiomas. Con esto haremos que su grado de consciencia intercultural aumente,

2. Con excepción de las tareas de brainstorming. 
habrán producido mucho output, habrán reforzado su competencia léxica y gramatical y todo ello nos habrá llevado al punto en que en la puesta en común el docente verbalizará aquello que en esa aula no se tolerará (comer, beber, llamar por teléfono mientras dura la clase, llevar gorra...) de acuerdo con las normas culturales y sociales que se consideren oportunas. Haremos un cierre de actividad prometiendo que vamos a respetar esas normas y que quizás habrá cosas que podamos llegar a hacer que puedan resultar ofensivas para otras personas, pero que no se hacen con mala intención. Si se considera necesario pueden hacerse constar de manera explícita en un mural en la pared, por ejemplo. Quizás esto sea más adecuado para un público más joven o que se sospeche particularmente conflictivo.

Esta es una posible sesión de introducción que sirve para trabajar diferentes aspectos como la dinámica de grupos, cuestiones lingüísticas, aspectos culturales y de normas sociales del aula. Como se puede observar se busca que los elementos interactúen de manera positiva. Otras opciones que algunos autores han dado para exteriorizar las normas es hacer contratos de aprendizaje donde el profesor se compromete a mantener una determinada actitud o tipo de docencia y el alumnado también y posteriormente se firma y se guarda. A esta tarea se le puede revestir de pompa para hacerlo más solemne y puede cerrarse con un apretón de manos que nos llevaría a otra cuestión intercultural que es cómo se manifiesta el acuerdo formal en diferentes culturas.

Por otra parte, en las primeras sesiones se debe promover la variedad de agrupaciones para promover la socialización al máximo y el conocimiento mutuo (Sousa, 2011: 79). Esto puede realizarse con técnicas externas como agruparlos con tarjetas, numerándolos, etc., pero no solo, ya que debemos combinarlas con reagrupaciones que también sean más significativas como, por ejemplo y ya lo hemos puesto en práctica en la actividad anterior, usando como elemento unificador temas de interés comunes. Esto desarrollará un sentimiento fuerte de cohesión y colaboración entre todas las personas del grupo (Wlodkowski, 1985: 206).

Otro ejemplo de sesión donde se abarcan varios componentes del proceso de enseñanza a aprendizaje es la siguiente. En el nivel A2 las rutinas son un contenido típico y que pueden trabajarse con un juego de "Y tú, ¿̇de qué eres?, ¿de Nesquik o de Colacao?" Probablemente no conozcan los productos, pero les podemos enseñar el vídeo del Comidista ${ }^{3}$. Se puede presentar como una tarea de comprensión auditiva y cultural, ya que está protagonizado por cocineros. Se les pide que en pequeños grupos que definan el desayuno más común en sus países,

3. https://elcomidista.elpais.com/elcomidista/2016/01/11/articulo/1452537563_192653.html Si se prefiere una comprensión lectora hay infinidad de textos adaptables: http://www.revistagq.com/la-buena-vida/ articulos/cola-cao-vs-nesquik/19539 
regiones o ciudades. Además de que comenten otras preguntas de un tono más informal sobre, por ejemplo, qué comen cuando tienen un mal día, por ejemplo, qué hacen para preparar un desayuno especial, cuándo los hacen... Se puede introducir también el tema de rutinas diarias con imágenes como las siguientes y que a través de ellas se hagan entrevistas sobre sus vidas, pero dándole un punto más lúdico y humorístico. Les proporcionamos una ficha con estas imágenes (o las proyectamos) y debatimos sobre ellas: qué creen que representan, creen que es verdad que el mundo se divide en esos dos tipos de personas y cuáles son, cuáles son las que les representan más y por qué, etc. (o les pedimos que se levanten y pongan pegatinas en las imágenes que más le representan y esto nos puede servir después para hacer la media de la clase y explicar otro contenido léxico: la mayoría, muchos...). Otra variante es que puede comenzar el docente contando sus "preferencias/manías" y explicando qué es lo más raro que hace. Ese ejemplo de exposición sincera y sin miedo a los prejuicios servirá de ejemplo para muchos de ellos que querrán compartir su experiencia vital también.

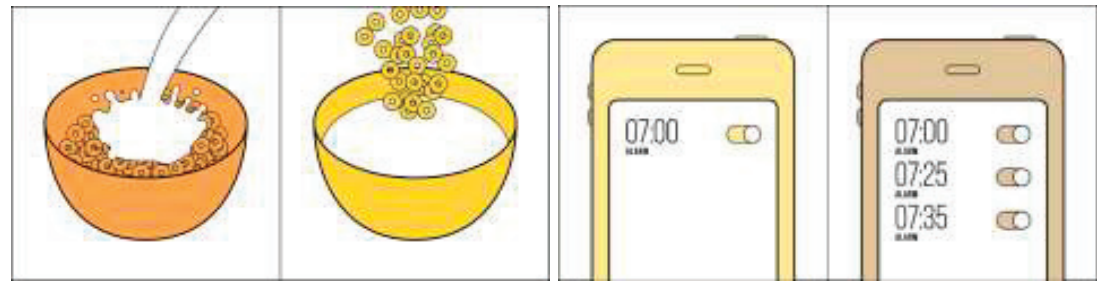

Ilustraciones 1 y 2 del artista Joao Rocha ${ }^{4}$

En resumen, consideramos que tomar una actitud proactiva en el surgimiento de una dinámica de grupo positiva es posible y necesaria, así como lo es que esta repercuta positivamente en otros elementos del sistema como, por ejemplo, la motivación.

\subsection{El desarrollo posterior del grupo}

Crear la filiación a un grupo es "relativamente fácil", ya que la gente comienza a conocerse (Hadfield, 1992: 46) y está predispuesta a ello por lo general, pero mantener la cohesión durante un semestre o año es más difícil. Hay que lograr conservar el contacto entre todos, desarrollar la cooperación, alentar la empatía, etc.

4. Las ilustraciones son del artista Joao Rocha: https://2kindsofpeople.tumblr.com/ 
Después de las primeras sesiones y el fuerte trabajo que se habrá realizado sobre el conocimiento mutuo, se deben seguir manteniendo los cambios frecuentes de modos de agrupación. Además, buscaremos que la máxima cantidad de tiempo pasado en el aula sea gratificante y que se promueva una valoración positiva entre ellos. También, haremos palpable el progreso alcanzado como grupo. Todo ello aderezado con leyendas de anécdotas y un toque de humor que garantice el buen ambiente emocional, siendo conscientes de que esto no ocurrirá de manera constante, sino que habrá oscilaciones propias de la variabilidad que implica que cada estudiante es único, pero consideramos que una fuerte dinámica de grupo puede ayudar a sobrellevar la presencia de desmotivadores puntuales como el cansancio, disgusto ante una actividad, etc.

Las reagrupaciones en esta etapa se pueden formular ya en torno a otros temas que no sean (solo) los intereses comunes o para conocerse, sino que el objetivo sea cooperar para trabajar en una tarea. Serían grupos informales de aprendizaje cooperativo (Richards y Rodgers, 2003: 193) que se forman solo para trabajar juntos durante una sesión, por ejemplo. Estos podrían hacerse a través, una vez más, de la posibilidad de elegir o siendo pautado por el docente. Se pueden plantear grupos que trabajen en torno a una destreza, por ejemplo, que en una zona trabajen con comprensión auditiva, en otra con comprensión lectora, etc. De este modo les damos la oportunidad de bien sentirse más cómodos en lo que más les gusta o dominan, bien de retarse a sí mismos para centrar sus esfuerzos en lo que les cueste más. Otra opción es que elijan, en general, un problema que resolver. Todo esto se puede plantear de modo que los materiales que tengan los diferentes grupos sean interdependientes para tener que hacer una puesta en común para lograr un éxito final conjunto. Se puede preparar de modo que la información sea interdependiente y luego se reagrupen de nuevo, pero intercalando la destreza, el problema, etc., es decir, uno de cada tema se iría de representante de ese tema a cada nuevo grupo formado y allí se haría la puesta en común. De este modo seguimos promoviendo la constante interacción social y la producción de output.

Ese tiempo durante el que trabajan juntos nos da pie a tomar nota de anécdotas graciosas que nos ofrezcan la oportunidad de crear "leyendas de grupo" que nos permitan ponernos nostálgicos en algún momento del curso y rememorar los buenos momentos porque las actividades que se diseñen deben ser eficaces cognitiva, social, cultural y emocionalmente (Méndez Santos, 2016a) para repercutir en el máximo número de elementos del sistema de aprendizaje con el fin de lograr el flujo. 
Dos propuestas concretas para que se perciba el progreso y para que la valoración positiva sea plena giran en torno a visibilizar el aprendizaje y los logros y, por otro lado, que la consecución de estos promueva sentimientos positivos.

Fomentar la percepción del progreso es una estrategia didáctica que se basa en considerar los aspectos motivacionales relacionados con la teoría del establecimiento de objetivos que describieron Locke y Latham (1990). Esta defiende que las acciones humanas vienen determinadas por un propósito y este debe ser palmariamente fijado, dado que ese establecimiento claro de objetivos incrementa la productividad. De hecho, en un estudio que resume Dörnyei (2008: 117) en su obra sobre la motivación nos explica que en un experimento con tres grupos que se hizo para comprobar la influencia del establecimiento de objetivos sobre la didáctica de matemáticas obtuvo unos resultados muy llamativos: aquel grupo que tuvo unas instrucciones claras y objetivos específicos a corto plazo logró completar al final de la cuarta sesión el 74\% de los materiales didácticos, mientras que el grupo cuyos objetivos eran distantes solo logró el 55\% y el último, con instrucciones vagas y objetivo difuso solo terminó el 53\%.

Esto tiene unas consecuencias prácticas claras. Los objetivos generales que nos hemos marcado al principio del curso pueden formar parte de un mural de clase y cada vez que se alcance uno, el alumnado puede proceder a marcarle un tic $(\sqrt{ })$. Si esto no considera factible de forma global -dependerá de la duración del curso-, puede realizarse para cada unidad que además cumplirá ese argumen-

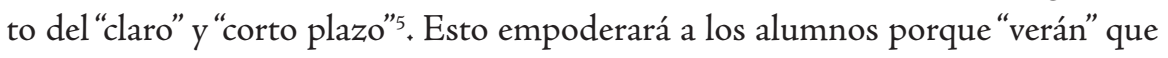
realmente están aprendiendo y mucho. Un truco práctico fácilmente realizable es comenzar cada sesión estableciendo en la parte derecha de la pizarra los objetivos del día. Deben ser acordes con el nivel y "fácilmente" alcanzables, para atender a las diferencias de nivel que quizás tengamos en el aula y para que ello no menoscabe la moral de aquellos no están por encima de la media. Al terminar la sesión se comprobará que esos mínimos que hemos establecido se han logrado. Si la sensación general fuese negativa, el docente ya tiene una herramienta más de evaluación de dominio continua que no requiere un examen de cada sesión.

Por otra parte, esta consecución de logros sobre la competencia comunicativa puede vincularse en las actividades con, por ejemplo, una valoración mutua positiva de los aprendices. Como se explica en Méndez Santos (2016b), la enseñanza de contenidos lingüísticos puede ser empleados para mejorar el ambiente afectivo de modo que se busque la integración y codependencia de diferentes aspectos del aprendizaje. En ese artículo se plantea una tarea para practicar los superlativos, las preposiciones, los adjetivos calificativos, la expresión de apreciación y cuestio-

5. Esta idea debe ser adaptada a cada contexto, ya que hay cursos por semanas, quincenas, meses, semestres... Si el curso es largo conviene más visualizarlos por unidades. 
nes culturales relacionadas con personajes famosos hispanohablantes. La parte final de la sesión consiste en que pongan en práctica aquello que han ido usando, pero en este momento ya de un modo más personal: deben preparar una banda de valoración para sus compañeros señalando en ella el aspecto más positivo del que le haya tocado o le hayamos asignado. La parte final de la sesión consiste en la entrega de las bandas y en un pequeño discurso de agradecimiento que deben dar. El nivel de clímax alcanzado es fuerte: se hacen fotos, se felicitan, se aplauden, etc. Su imagen social es muy buena. En todas las ocasiones que se ha puesto en práctica esta actividad se consiguen unos resultados muy alentadores tanto sobre los contenidos lingüísticos, como sobre su autoestima y la cohesión grupal.

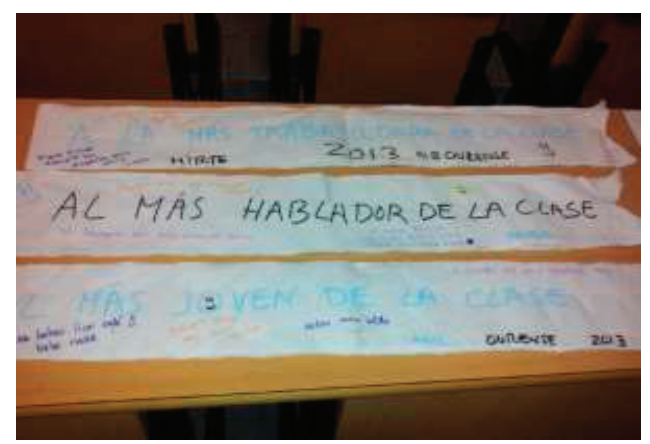

Imagen 1: Actividad sobre el superlativo

En este momento de desarrollo del grupo se pueden, también, localizar ya "amenazas comunes" o "dificultades compartidas" (Dörnyei, 2008: 71), dado que esto genera la solidaridad de compañeros de fatigas. Una forma práctica de operativizar esto en el aula podría ser usar esa dificultad compartida como elemento de conexión como, por ejemplo, haciendo el club de fans de $x$ participio irregular y ese día ese grupo de personas trabajará de modo conjunto. Sabemos que la instrucción explícita mejora los resultados de aprendizaje de la gramática (Long, 1983; Cadierno, 2010) y con este tipo de actividades se realza el input sobre el que queremos poner el esfuerzo cognitivo de manera selectiva, con un punto divertido y emocional para que también tenga consecuencias en la dinámica de grupo. 


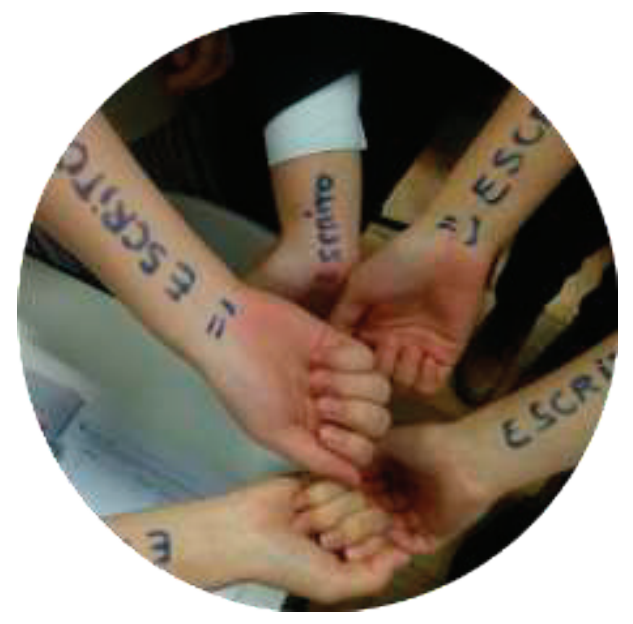

Imagen 2: Club de las estudiantes que amaban el participio irregular "escrito". Primer curso de español en la Universidad Prefectural de Aichi (Japón)

La competición puede ser usada como factor de cohesión cuando un grupo ya está muy seguro de sí mismo, aunque con precaución porque se puede producir una conducta defensiva. Planteada de una forma divertida, es decir, no vinculada con quien sabe más o quien sabe menos, por ejemplo, puede servir para darle un toque de ludicidad al ambiente y siempre asegurándonos de que los "perdedores" no se lo tomen demasiado en serio (Dörnyei, 2008: 110).

Un último aspecto que cabe destacar y que ya hemos citado en diversas ocasiones es que la interacción debe ser significativa y, además, siempre que sea posible que dé pie a compartir información personal genuina, que no tiene que ser necesariamente privada ${ }^{6}$. Siempre sin olvidar que lo que es cultural y emocionalmente significativo puede no serlo para otra (Ginsberg y Wlodkowski, 2000:3), en otras palabras, hay que ser conscientes de que en ciertas sociedades hay temas que son tabú.

\subsection{La disolución del grupo}

Tan importante como el inicio y el desarrollo del curso y la dinámica de grupo que se establece, es preparar el final correcto. El alumnado ha compartido unos días, unas semanas o meses de vivencias compartidas que habrán dado mucho de sí. Conviene que la última sesión se dedique a hacer una retrospección de lo vivido y aprendido. Un buen (mal) recuerdo puede comprometer nuestra per-

6. Entendiendo privado como algo restringido a un grupo íntimo y limitado de personas (Diccionario Clave, en línea: "Que pertenece o está reservado a una sola persona o a un número limitado y escogido de personas"). 
severancia a la hora de continuar estudiando una lengua extranjera. Este es un proceso largo y arduo, así que conviene que en cada paso haya una sensación de éxito académico y emocional que refuerce el compromiso.

Una actividad que se puede llevar a cabo al final del curso para crear una despedida calurosa y afectuosa es una "caja de la memoria" (o sobre, en nuestro caso). En él, cada estudiante deposita mensajes y un objeto personal que le represente. Todos dejarán algo en el "sobre/caja" de todos. Luego el docente lo cerrará/ lacrará/precintará y se promete que cuando se abra, al cabo de unos días, unas semanas o unos meses, deben notificarlo al grupo a través del foro que se haya usado durante el curso (blog, redes sociales, plataforma educativa...). En ese momento deberán explicar qué han sentido al abrirlo y qué emociones recuerdan del curso. Esta forma de crear impactos emocionales compartidos es altamente motivadora.

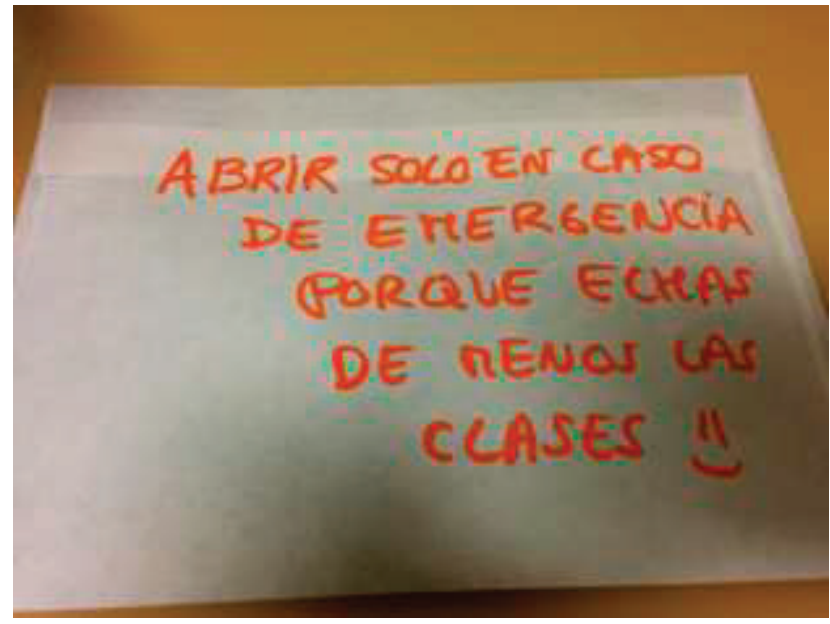

Imagen 3: Sobre de la memoria del grupo de A2. Centro de Linguas de la Universidade de Vigo (España).

Otra opción es crear una caja de los buenos momentos durante el curso. En ella cada vez que ocurra algo curioso, anecdótico, divertido, raro... se debe depositar una nota. La caja se abrirá en las últimas clases y servirá como elemento creador y mantenedor de las leyendas de grupo creadas. Cuando se abra y se revisen se contarán las historias con lo que será un momento ideal, además, para repasar los contenidos lingüísticos relacionados con la narración de hechos pasados. En un nivel B1 sería ideal para abrir hacia mitad de curso que es cuando ya se han estudiado los contenidos e, incluso, si se considera adecuado se puede tomar 
como muestras para revisar los errores que antes se cometían y trabajar con ellos para comprobar si se han superado.

\section{Conclusiones}

La enseñanza formal de lenguas extranjeras es un proceso exigente que demanda un fuerte esfuerzo cognitivo y emocional. Por ello, es necesario que el docente sea consciente y reflexione sobre todos los elementos presentes durante el proceso de enseñanza-aprendizaje y las relaciones de mutua influencia que se establecen entre ellos. En este trabajo hemos querido reflexionar más concretamente sobre uno, la dinámica de grupos, y las conexiones de interdependencia que establece con otros factores implicados.

Así, al entender que un ser humano es social y que aprende en interacción con los demás, el rol de las personas con las que nos topamos en el aula cobra una vital importancia y se torna significativo. Por consiguiente, planificar y gestionar el grupo es fundamental. Un ambiente emocional positivo no se logra solo por casualidad, sino que puede ser el resultado de un trabajo proactivo, consciente y sistemático.

Este compromiso didáctico debe tener en cuenta todos los aspectos sobre los que la gestión de grupo puede repercutir, ya que sabemos que una buena dinámica favorece una mayor presencia de input comprensible, así como una mayor producción de output. Además, también se reduce el nivel de ansiedad, aumenta el grado de motivación y cooperación y se logra que los discentes tengan una imagen social y autoestima más vigorosas. Esta suma de factores desemboca en una mayor cantidad de aprendizaje y de mejor calidad, ya que la experiencia vivida es positiva. Este aspecto puede tener una influencia directa en la perseverancia necesaria para continuar el aprendizaje de la lengua en siguientes cursos o a lo largo de la vida.

Las estrategias didácticas concretas que hemos descrito con las que operativizar en el aula estas consideraciones teóricas son las siguientes. Durante los primeros días, se debe crear una fuerte filiación usando muchas actividades de conocimiento mutuo y cambios de agrupación constantes que tengan como nexo intereses temáticos comunes. Además, se buscará una reducción de cualquier situación que amenace la imagen pública como, por ejemplo, la corrección personal de errores, o el uso de competiciones de dominio que influyan negativamente sobre la percepción de autoeficacia del alumnado. Se establecerán las normas del grupo y con ellas se prestará atención al respeto y el conocimiento intercultural 
que es fundamental en grupos heterogéneos para evitar o reducir el impacto de las posibles ofensas.

Durante el desarrollo del grupo, se debe seguir manteniendo la reagrupación frecuente, pero esta se puede lograr a través de otras técnicas como que puedan elegir el problema que tratan, la destreza que entrenan, etc. Asimismo, se promoverá que los contenidos lingüísticos se vinculen a actividades que promuevan la valoración mutua para aumentar la autoestima de cada individuo y la filiación grupal. Se usarán las anécdotas como elementos cohesionadores a modo de leyendas y se puede utilizar alguna dificultad específica, por ejemplo, como dificultad compartida que genere un sentimiento de camaradería.

Tan importante como el inicio y el desarrollo del grupo es su disolución, ya que una retrospectiva positiva incrementa la posibilidad de que el alumnado continúe aprendiendo la lengua. Para ello se pueden aprovechar los "mejores momentos" del curso como hilo conductor de una actividad de despedida y preparar otra que se complete fuera del aula y en diferido porque puede alargar la vida del impacto emocional positivo de haber formado parte de ese grupo y de esa experiencia de aprendizaje.

En suma, la confluencia de los múltiples factores, incluida la dinámica de grupos, que se ven implicados durante la enseñanza y aprendizaje de una lengua constituyen un sistema dinámico complejo en el que todos se influyen entre sí y van adaptándose. Un desarrollo óptimo de un equilibrio entre ellos puede crear las condiciones necesarias para alcanza el flujo y, por ende, un aprendizaje exitoso en todos los niveles social, cultural, cognitivo y emocional.

\section{Referencias bibliográficas}

Bandura, A. (I997). Self-efficacy: The Exercise Control. Nueva York: Freeman.

Byram, M. y Fleming, M. (200I). Perspectivas interculturales en el aprendizaje de idiomas: Enfoques a través del teatro y la etnografía. Madrid: Edinumen.

Cadierno, T. (2010). «El aprendizaje y la enseñanza de la gramática en el español como segunda lengua». MarcoELE, 10, 1-18. Recuperado de https:// marcoele.com/el-aprendizaje-de-la-gramatica/ [Fecha de consulta: 5 de mayo de 2018]

Carrera, B.y Mazzarella, C. (2001)+ «Vigostky. Enfoque sociocultural»+Educere, 13, 41-44. Recuperado de http://www.redalyc.org/html/356/35601309/ [Fecha de consulta: 5 de mayo de 2018] 
Clement, R+; Dörnyei, Z. y Noels, K. (i994)+ «Motivation, Self-Confidence, and Group Cohesion in Foreign Language Classroom». Language Learning, $44 \div 3,417-448$.

Covington, M. V. (I998). The will to learn. A guide for motivating young people. Cambridge: Cambridge University Press.

Csíkszentminályi, M. (20I4). Fluir. Una psicología de la felicidad. Barcelona: DeBolsillo.

Deci, E. L*y Ryan, R. M* (1985). Intrinsic motivation and self-determination in buman behavior. Nueva York: Plenum

De Santiago Guervós, J. y Fernández González, J. (2017). Fundamentos para la enseñanza del español como 2/L. Madrid: Arco/Libros.

Dörnyei, Z. y Malderez, A. (2000). «El papel de la dinámica de grupos en el aprendizaje y la enseñanza de lenguas extranjeras». En J. Arnold (Ed.). La dimensión afectiva en el aprendizaje de idiomas. Madrid: Cambridge University Press.

Dörnyei, Z. y Otтo, I. (1998). «Motivation in action: A process model of L2 motivation». Working papers in Applied Linguistics, 4, 43-69.

Dörnyei, Z. (2001). Teaching and researching motivation. Edimburgo: Pearson.

Dörnyei, Z. (2008). Estrategias de motivación en el aula de lenguas. Barcelona: UOC.

Dörnyei, Z. (2009). «Motivation in second and foreign language learning»+ Language Teaching, 31, (3), 117-135.

Dörnyei, Z.; MacIntrre, P. D. y Henry, A. (2015). «Introduction: Applying Complex Dynamic Systems Principles to Empirical Research on L2 Motivation». En Dörnyei, Z.; MacIntrye, P. D. y Henry, A. (Eds.). Motivational Dynamics in Language Learning. Bristol: Multilingual Matters.

Ehrman, M. E. y Dörnyei, Z. (1998). Interpersonal dynamics in second language education: The visible and invisible classroom. California: Sage.

Gardner, R.C. y Lambert, W.E. (1972). Attitudes and Motivation in Second Language Learning. Rowley: Newbury House.

Gass, S. M. y Mackey, A. (2015). «Input, Interaction, and Output in Second Language Learning». En B. VanPatten y J. Williams (Eds.). Theories in Second Language Learning. An introduction. Nueva York: Routledge.

Ginsberg, M. B. y Whodkowski, R. (2000). Creating Highly Motivating Classrooms for all students. San Francisco: Jossey-Bass.

Goleman, D. (2014): Liderazgo. El poder de la inteligencia emocional. Barcelona: B de Bolsillo.

Hadfield, J. (1992). Classroom dynamics. Oxford. Oxford University Press. 
Hiver, P. (2015). «Attractor states». En Z. Dörnyei, P. MacIntyre y A. Henry (Eds.). Motivational dynamics in language learning. Bristol: Multilingual Matters.

Horwitz, E. K. (2017). «On the misreading of Horwitz, Horwitz and Cope (1986) and the Need to Balance Anxiety Research and the Experiences of Anxious Language Learners». En C. Gkonou, M. Daubney y J.M. Dewaele (Eds.). New insights into Language Anxiety. Theory, Research and Educational implications. Bristol: Multilingual Matters.

Instituto Cervantes (2012). Las competencias clave del profesorado de lenguas segundas y extranjeras. En línea: https://cvc.cervantes.es/ensenanza/biblioteca_ ele/competencias/default.htm [Fecha de consulta: 5 de mayo de 2018]

Kiкuchi, K. (2015). Demotivation in Second Language Learning. Insights from Japan. Bristol: Multilingual Matters.

King, J. y Smith, L. (2017). «Social Anxiety and Silence in Japan's Tertiary Foreign Language Classrooms». En C. Gkonou, M. Daubney y J.M. Dewaele (Eds.). New insights into Language Anxiety. Theory, Research and Educational implications. Bristol: Multilingual Matters.

Larsen-Freeman, D. (2015). «Ten Lessons from Complex Dynamic Systems Theory: What is on offer». En Z. Dörnyei, P.D. MacIntyre y H. Alastair (Eds.). Motivational Dynamics in Language Learning. Bristol: Multilingual Matters.

Locke, E.A. y Latham, G. P. (1990). Volition and Personality: Action versus State Orientation. Seattle: Hogrefe and Huber.

Long, M. (1983). «Does Second Language Instruction Make a Difference? A review of Research». TESOL Quaterly, 17 (3), 359-382.

MacIntyre, P. D. (2017). «An overview of Language Anxiety Research and Trends in its Development». En C. Gkonou, M. Daubney y J.M. Dewaele (Eds.). New insights into Language Anxiety. Theory, Research and Educational implications. Bristol: Multilingual Matters.

Martín Perís, E. (Coord.) (2008). Diccionario de términos clave de ELE. Recuperado de https://cvc.cervantes.es/ensenanza/biblioteca_ele/diccio_ele/default. htm [Fecha de consulta: 5 de mayo de 2018]

Méndez Santos, M. C. (2016a). «Learning by feeling: sentimenduen bidetik ikasi. Gerora gogoratuko diren jardueren diseinu eraginkorra, atzerriko hizkuntzen (AH) eskolan», E-Hizpide, 89, 1-23.

Méndez Santos, M. C. (2016b). «Gramática afectiva en contextos de instrucción formal de español como lengua extranjera (ELE)», Hesperia. Anuario de Filología Hispánica, XIX (2), 51-84. 
Mercer, S. (2015). «Social network analysis and complex dynamic systems». En Z. Dörnyei, P. MacIntyre y A. Henry (Eds.). Motivational dynamics in language learning. Bristol: Multilingual Matters.

Oblitas, L+; Martínez Sánchez, F. y Palmero, F. (2008). «Emociones y salud». En F. Palmero y F. Martínez Sánchez (Eds.). Motivación y emoción. Madrid: McGrawHill.

Oxford, R.L. (2000). «La ansiedad y el alumno de idiomasः nuevas ideas». En J. Arnold (Ed.). La dimensión afectiva en el aprendizaje de idiomas. Madrid: Cambridge University Press.

Richards, J. C. y Lockhart, C. (1998). Estrategias de reflexión sobre la enseñan$z a$ de idiomas. Madrid: Edinumen.

Richards, J. C. y Rodgers, T. S. (2003). Enfoques y métodos en la enseñanza de idiomas. Madrid: Cambridge University Press.

Sousa, D. (2011). How the brain learns. California: Corwin.

Stevick, E. (1980). Teaching languages. A Way and Ways. Rowley: Newbury.

Swain, M. (1993). «The Output Hypothesis: Just Speaking and Writing aren't enough». Canadian Modern Language Learning, 50, 158-164.

Weiner, B. (1992). Human motivation: Metaphors, Theories and Research. Newbury: Sage.

Williams, M. y Burden, R.L. (2008). Psicología para profesores de idiomas. Madrid: Edinumen (Edición en inglés de 1997).

Wlodkowski, R. (1985). Enhancing adult motivation to learn. San Francisco: Jossey-Bass. 